\title{
AMBIENTES MICELARES EM QUÍMICA ANALÍTICA
}

\author{
Nelson Maniasso
}

Centro de Energia Nuclear na Agricultura, Universidade de São Paulo, CP 96, 13400-970 Piracicaba - SP

Recebido em 20/12/99; aceito em 18/4/00

\begin{abstract}
MICELLAR MEDIA IN ANALYTICAL CHEMISTRY. This review deals with the general use of the surfactants in Analytical Chemistry. Principal characteristic of the micelle is the improvement in selectivity and/or sensitivity of the analytical determination with emphasis on the catalytic reaction and "cloud point" extraction.
\end{abstract}

Keywords: micellar media; cloud point extraction; catalytic reactions.

\section{INTRODUÇÃO}

Tensoativos (surfactantes*) são importantes em Química Analítica devido, principalmente, à sua capacidade em modificar algumas propriedades reacionais com consequente melhoria em sensibilidade e/ou seletividade analítica. As principais características do uso de tensoativos estão relacionadas à formação de ambientes organizados, também conhecidos como ambientes micelares ${ }^{1}$.

Nas últimas décadas, o uso de tensoativos teve um aumento significativo em praticamente todos os campos da Química Analítica, devido a suas características em modificar diferentes propriedades reacionais associadas aos crescente emprego destes compostos nos mais variados produtos de forma natural ou sintética ${ }^{1-4}$.

Os tensoativos são frequentemente empregados para modificar o meio reacional permitindo solubilizar espécies de baixa solubilidade ou promover um novo meio que pode modificar a velocidade reacional, a posição de equilíbrio das reações químicas e em alguns casos a estereoquímica destas dependendo da natureza da reação, do tipo de reativo (eletrofílico, nucleofílico, etc) e do tipo e forma (catiônica, aniônica, etc) da micela ${ }^{1-4}$.

Pode-se destacar o emprego de ambientes micelares principalmente sob dois aspectos. O primeiro se refere à exploração das características do ambiente micelar, formado no meio reacional para a melhoria da sensibilidade e/ou seletividade, com ênfase a reações catalíticas, e o segundo, se relaciona a etapas de concentração e/ou separação, empregando tensoativos em substituição às metodologias tradicionais (extração líquido-líquido, troca iônica) pela separação em duas fases isotrópicas, fenômeno este denominado "cloud point". A aplicabilidade do meio micelar pode ser observada nos trabalhos de Pelizzetti e Pramauro ${ }^{4}$ e Quina e Hinze ${ }^{5}$, para compostos inorgânicos e orgânicos.

A ampla utilização dos tensoativos em óleos para automóveis, na prospecção de petróleo, em fármacos, em produtos domésticos tais como xampus, suavizantes, condicionadores, detergentes, cosméticos, etc ${ }^{1,2}$ são indicativos de sua versatilidade.

Os problemas iniciais relacionados ao uso de tensoativos nos mais diferentes produtos eram relativos ao emprego de compostos não biodegradáveis, os quais proporcionavam sérios problemas de contaminação ao meio ambiente. Para solucionar estes inconvenientes, novos tensoativos biodegradáveis

e-mail maniasso@cena.usp.br

* A definição da palavra surfactante é baseada na contração da frase em inglês que descreve "surface-active agents". Estes possuem uma superfície ativa, devido à concentração de determinadas espécies em uma região interfásica : ar-água, óleo-água ou sólido líquido. denominados "produtos verdes", foram desenvolvidos. O desenvolvimento destes novos produtos associados aos já existentes propiciaram um incremento do uso dos mesmos em Química Analítica.

Um tensoativo típico possui a estrutura $\mathrm{R}-\mathrm{X}$, onde $\mathrm{R}$ é uma cadeia de hidrocarboneto variando de $8-18$ átomos (normalmente linear) e X é o grupo cabeça, polar (ou iônico). Dependendo de $\mathrm{X}$, os tensoativos podem ser classificados como nãoiônicos, catiônicos, aniônicos ou anfóteros ${ }^{4}$.

Um tensoativo catiônico possui em geral a formula $\mathrm{R}_{\mathrm{n}} \mathrm{X}^{+} \mathrm{Y}^{-}$ , onde $\mathrm{R}$ representa uma ou mais cadeias hidrofóbicas, $\mathrm{X}$ é um elemento capaz de formar uma estrutura cationnica e Y é um contra íon. Em princípio, X pode ser N, P, S, As, Te, Sb, Bi e os halogênios ${ }^{6,7}$.

Dentre os tensoativos aniônicos mais frequentemente utilizados, estão aqueles que possuem sais de ácidos carboxílicos (graxos) monopróticos ou polipróticos com metais alcalinos ou alcalinos terrosos, ácidos como sulfúrico, sulfônico e fosfórico contendo um substituinte de hidrocarboneto saturado ou insaturado ${ }^{8}$.

Para os anfóteros (os quais possuem ambos grupos aniônicos e catiônicos no meio hidrofóbico), e dependendo do $\mathrm{pH}$ da solução e da estrutura, pode prevalecer a espécie aniônica, catiônica ou neutra. Os tensoativos anfóteros mais comuns incluem $\mathrm{N}$-alquil e $\mathrm{C}$-alquil betaina e sultaina como também álcool amino fosfatidil e ácidos ${ }^{9}$.

Os tensoativos não-iônicos são derivados do polioxietileno e polioxipropileno (de compostos com alquil fenol e álcool, esteres de ácidos graxos, alquilaminas, amidas e mercaptanas) ou polialcoóis, esteres de carboidratos, amidas de álcoois graxos e óxidos de amidas graxas ${ }^{10}$.

A Tabela 1, indica os principais agentes tensoativos empregados para o estabelecimento de ambientes organizados visando melhoria de desempenho em métodos analíticos.

Micelas são agregados moleculares, possuindo ambas as regiões estruturais hidrofílica e hidrofóbica, que dinamicamente se associam espontaneamente em solução aquosa a partir de certa concentração crítica (CMC), formando grandes agregados moleculares de dimensões coloidais, chamados micelas. Abaixo da CMC, o tensoativo está predominantemente na forma de monômeros; quando a concentração está abaixo, porém próxima da CMC, existe um equilíbrio dinâmico entre monômeros e micelas ${ }^{3}$ (Figura 1). A combinação destas propriedades distintas confere à molécula características únicas na dissolução aquosa ${ }^{1-5}$. Em concentrações acima da CMC, as micelas possuem um diâmetro entre 3-6 $\mathrm{mm}$ o que representa de 30-200 monômeros. A CMC depende da estrutura do tensoativo (tamanho da cadeia do hidrocarboneto) e das condições experimentais (força iônica, contra-íons, temperatura, etc). 
Tabela 1. Agentes tensoativos de uso comum em Química Analítica.

\begin{tabular}{|c|c|c|}
\hline TIPO & AGENTE TENSOATIVO & FORMULA \\
\hline \multirow[t]{3}{*}{ CATIÔNICOS } & $\begin{array}{l}\text { Brometo de cetiltrimetil amônio } \\
\text { (CTAB) }\end{array}$ & $\mathrm{CH}_{3}\left(\mathrm{CH}_{2}\right)_{15} \mathrm{~N}^{+}\left(\mathrm{CH}_{3}\right)_{3} \mathrm{Br}^{-}$ \\
\hline & $\begin{array}{l}\text { Brometo de dodeciltrimetil amônio } \\
\text { (DTAB) }\end{array}$ & $\mathrm{CH}_{3}\left(\mathrm{CH}_{2}\right)_{11} \mathrm{~N}^{+}\left(\mathrm{CH}_{3}\right)_{3} \mathrm{Br}^{-}$ \\
\hline & $\begin{array}{l}\text { Cloreto de cetilpiridino } \\
\text { (CICP) }\end{array}$ & $\mathrm{CH}_{3}\left(\mathrm{CH}_{2}\right)_{15} \mathrm{~N}+\mathrm{Cl}^{-}$ \\
\hline \multirow[t]{3}{*}{ ANIÔNICOS } & Dodecil sulfato sódico (SDS) & $\mathrm{CH}_{3}\left(\mathrm{CH}_{2}\right)_{11} \mathrm{SO}_{4}^{-} \mathrm{Na}^{+}$ \\
\hline & $\begin{array}{l}\text { Bis(2-etilhexil) sulfosuccinato } \\
\text { sódico (Aerosol OT) }\end{array}$ & {$\left[\mathrm{CH}_{3}\left(\mathrm{CH}_{2}\right)_{3} \mathrm{CH}\left(\mathrm{C}_{2} \mathrm{H}_{5}\right) \mathrm{CH}_{2} \mathrm{OCO}\right]_{2} \mathrm{CHSO}_{3}{ }^{-} \mathrm{Na}^{+}$} \\
\hline & Dihexadecil fosfato (DHF) & {$\left[\mathrm{CH}_{3}\left(\mathrm{CH}_{2}\right)_{15}{ }^{\mathrm{O}}\right]_{2} \mathrm{PO}_{2}^{-}$} \\
\hline \multirow[t]{2}{*}{ NÃO IÔNICOS } & $\begin{array}{l}\text { Polioxietileno }(9-10) \text { p-tercotil } \\
\text { fenol (Triton X-100) }\end{array}$ & $\begin{array}{c}\left(\mathrm{CH}_{3}\right)_{3} \mathrm{C}\left(\mathrm{CH}_{2}\right) \mathrm{C}\left(\mathrm{CH}_{3}\right)_{2} \mathrm{O} \\
\left(\mathrm{OCH}_{2} \mathrm{CH}_{2}\right)_{23} \mathrm{OH}\end{array}$ \\
\hline & $\begin{array}{l}\text { Polioxietileno (23) } \\
\text { dodecanol (brij 35) }\end{array}$ & $\mathrm{CH}_{3}\left(\mathrm{CH}_{2}\right)_{11}\left(\mathrm{OCH}_{2} \mathrm{CH}_{2}\right)_{23} \mathrm{OH}$ \\
\hline \multirow[t]{2}{*}{ ANFÓTEROS } & $\begin{array}{l}\text { 3-(dodecildimetil amônio) propano } \\
\text { 1-sulfato (SB-12) }\end{array}$ & $\mathrm{CH}_{3}\left(\mathrm{CH}_{2}\right)_{11} \mathrm{~N}^{+}\left(\mathrm{CH}_{3}\right)_{2}\left(\mathrm{CH}_{2}\right)_{3} \mathrm{OSO}_{3}^{-}$ \\
\hline & $\begin{array}{l}\text { 4-(dodecildimetil amônio) } \\
\text { butirato (DAB) }\end{array}$ & $\mathrm{CH}_{3}\left(\mathrm{CH}_{2}\right)_{11} \mathrm{~N}^{+}\left(\mathrm{CH}_{3}\right)_{2}\left(\mathrm{CH}_{2}\right)_{3} \mathrm{COO}^{-}$ \\
\hline
\end{tabular}

As micelas são termodinamicamente estáveis e facilmente reprodutíveis, são destruídas pela diluição com água quando a concentração do tensoativo ficar abaixo da $\mathrm{CMC}^{4}$.

$\mathrm{O}$ processo de formação dos agregados ocorre num intervalo pequeno de concentrações, e pode ser detectado pela variação brusca produzida em determinadas propriedades físicoquímicas da solução em função da concentração do tensoativo como a tensão superficial, pressão osmótica e condutividade (só para tensoativos iônicos) ${ }^{11}$. Na Figura 2, é representada a variação de algumas propriedades e nela pode-se observar a mudança de comportamento que as mesmas experimentam ao alcançar a $\mathrm{CMC}^{12}$.

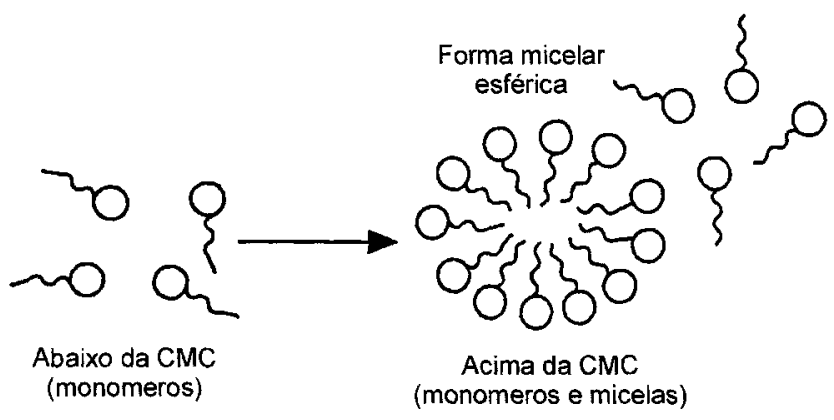

Figura 1. Formação do agregado micelar.

O termo "interface" indica o limite entre as duas fases imiscíveis, e o termo "superfície" indica uma interface onde uma fase é liquida e a outra é gasosa, geralmente ar. A quantidade mínima de trabalho para criar a interface é chamada de energia interfacial livre, medida por unidade de área, quando a tensão superficial entre as duas fases é determinada.

O tensoativo tem uma característica de estrutura molecular, consistindo de um grupo funcional que tem pequena atração pelo solvente chamado grupo liofílico. Este é conhecido como uma estrutura anfipática. Quando o tensoativo é dissolvido em um solvente, a presença do grupo liofóbico no interior do solvente causa uma distorção da estrutura líquida do solvente, aumentando a energia livre do sistema.

Em solução aquosa com tensoativo, esta distorção da água pelo grupo liofóbico (hidrofóbico) do tensoativo resulta no

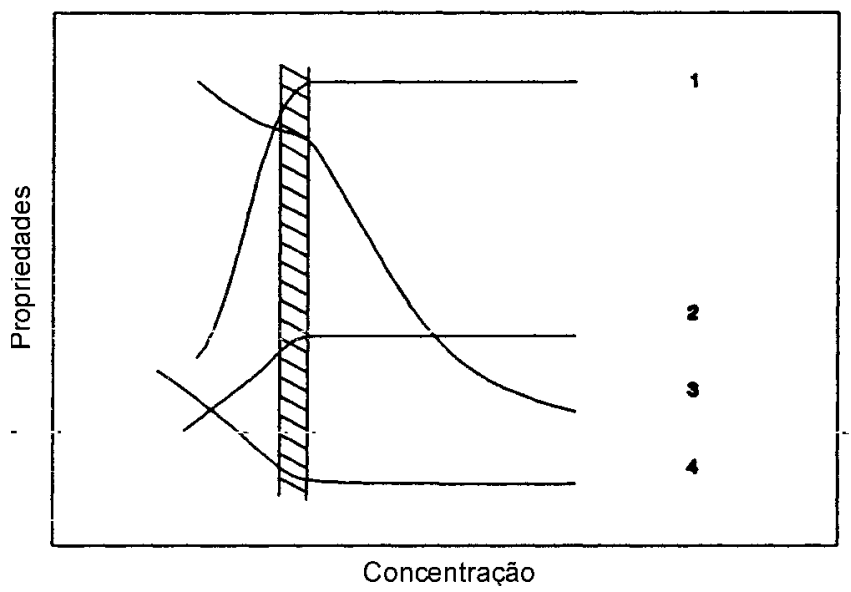

Figura 2. Variação de algumas propriedades físico-químicas. 1) Detergência, 2) Pressão osmótica, 3) Condutividade equivalente, 4) Tensão superficial, em função da concentração do tensoativo. A área hachureada corresponde à CMC.

aumento da energia livre do sistema. Quando este é dissolvido, o trabalho necessário para trazer uma molécula surfactante para a superfície é menor do que aquele relativo a uma molécula de água. A presença do tensoativo diminui o trabalho necessário para criar uma unidade de área de superfície (superfície de energia livre ou tensão superficial ${ }^{12}$.

\section{Tensoativos naturais e sintéticos}

Tensoativos são compostos anfifílicos, orgânicos ou organometálicos que formam colóides ou micelas em solução. Substâncias anfifílicas ou anfílicas são moléculas possuidoras de regiões distintas e características como hidrofóbicas e hidrofílicas. Como nestas substâncias apenas a polaridade das diferentes regiões varia enormemente, as mesmas são também denominadas de moléculas anfipáticas, heteropolares ou polarnão polares ${ }^{13,14}$.

Os tensoativos naturais incluem lipídeos simples (p. ex. ésteres de ácido carboxilílico), lipídeos complexos (esteres de ácidos graxos contendo fósforo, base nitrogenadas, e/ou açúcar) e ácidos bílicos tais como ácido cólico e deoxicólico. 
Contudo, com outras preparações bioquímicas, o isolamento do produto homogêneo puro frente a uma estrutura homóloga e isômera é extremamente difícil, especialmente para grandes quantidades de material. Além disso, a estrutura do lipídeo pode ser alterada durante sua extração e purificação ${ }^{15}$. Já para os tensoativos sintéticos estes problemas são minimizados, pois dependem da estrutura química das ligações como a porção hidrofóbica

\section{Formação das micelas}

Micelas não são estáticas, elas existem dentro de uma dinâmica de equilíbrio, simplesmente como um agregado dinâmi$\mathrm{co}^{14}$. A cinética das dissociações micelares foram medidas para vários tensoativos, em experimentos envolvendo parada de fluxo (stopped flow) $)^{4,16}$, salto de temperatura ${ }^{17,18}$, técnica de relação ultra-sônica ${ }^{19,20}$, etc.

Estes agregados podem participar de numerosas reações nas quais a solubilização de um ou mais reagentes na micela leva a uma significativa alteração na cinética reacional. A solubilização introduz duas novas situações que podem influenciar a velocidade reacional, alterar o local de distribuição do soluto (reagente) e da superfície (efeito interfacial) ${ }^{3}$. Para solutos neutros (hidrofóbicos), o efeito de velocidade na reação térmica bimolecular é normalmente tratado em termos do "modelo da pseudofase". Este modelo trata micelas ou outro meio coloidal como reações distintas ${ }^{3}$.

Cada micela é composta por um certo número de moléculas de tensoativo, denominado como número de agregação, que rege geralmente o tamanho e a geometria do sistema micelar ${ }^{21}$. O termo "micela normal" é utilizado para se referir a agregados de tensoativos em meio aquoso, o qual é apresentado na Figura 3.

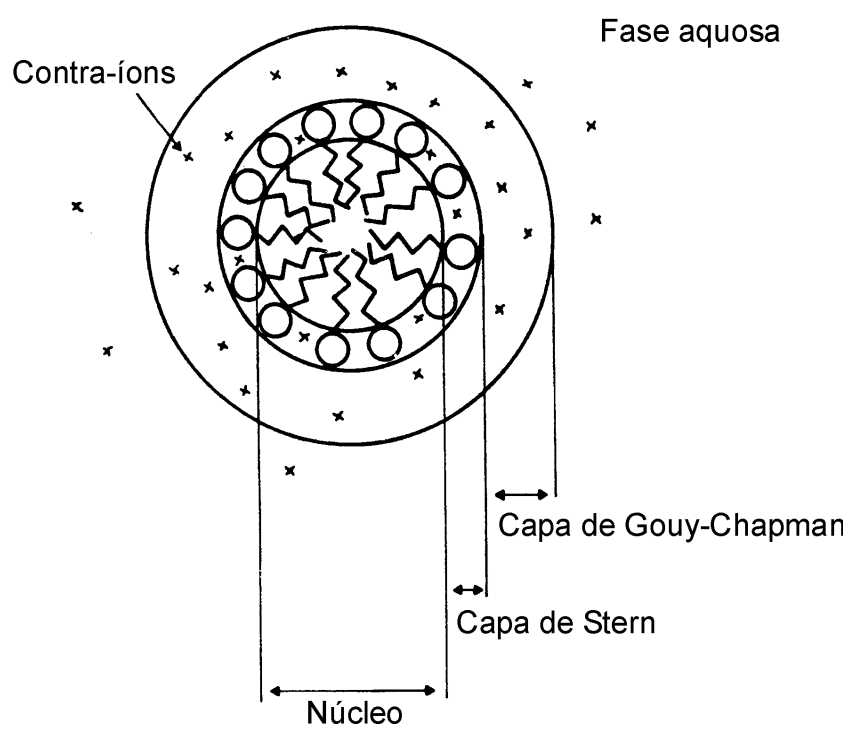

Figura 3. Representação bidimensional das regiões que formam uma micela iônica normal com estrutura esférica ${ }^{8} . X, O$ e $h$ indicam as localizações relativas dos contra-íons, do grupo cabeça, e das caudas (hidrocarbonetos), respectivamente.

A estrutura da micela normal formada indica que o grupo cabeça hidrofílico está direcionado para o contato com a solução aquosa formando uma superfície polar, enquanto que a cadeia linear (cauda) está em sentido inverso ao da água, formando um núcleo central não polar ${ }^{14}$.

A formação de associações de colóides pode também ocorrer em vários solventes não-polares; neste caso, os agregados dos tensoativos são denominados "micelas reversas" ou "micelas invertidas"14,22. Nos sistemas de micelas reversas, as cabeças polares dos anfifílicos estão concentradas no interior do agregado e por esta razão formam um núcleo central hidrofílico.

Uma propriedade importante das micelas é o seu poder de solubilizar os mais variados solutos ou espécies pouco solúveis. A quantidade de soluto solubilizada é em geral diretamente proporcional à concentração do tensoativo, desde que a concentração do tensoativo seja igual ou superior que a CMC e que existam várias possibilidades de solubilização no sistema micelar ${ }^{23}$.

Estes efeitos são consequência da solubilização dos reagentes na micela, sendo deste modo atribuídos ao balanço das interações de hidrofobicidade e eletrostática ocorrendo entre os reagentes e o sistema micelar.

Para várias reações, aspectos eletrostáticos simples podem ser considerados ao se explicar os efeitos micelares observados. Isto é esperado porque a taxa da reação nucleofilica que envolve um extrato solubilizado neutro e um nucleófilo pode ser acelerada por uma micela catiônica e inibida por uma micela aniônica. Efeito micelar oposto pode ser esperado para uma reação eletrofílica envolvendo um substrato e um eletrófilo. Quando sistemas envolvendo micelas não iônicas ou anfóteras são empregados, somente pequenos efeitos na cinética reacional são esperados.

Em um senso qualitativo, estes efeitos podem ser orientados em termos de estabilização eletrostática do estado de transição relativo para o conhecimento geral dos estados dos reagentes. Deve-se salientar que nem todos os incrementos ou inibição da reação podem ser explicados pela simples consideração eletrostática. Em vários casos, a interação hidrofóbica tem mais valor do que o desfavorável efeito eletrostático sendo necessário levá-la em consideração ${ }^{24}$.

Em contraste com a micela normal, o efeito catalítico das micelas reversas possue um conjunto mais complicado envolvendo outros fatores em adição ao eletrostático e a consideração hidrofóbica. A comparação das características de diferentes tipos de agregados que formam os agentes tensoativos podem se visualizados na Tabela 2 .

\section{Efeito micelar sobre as reações químicas: catálise micelar}

Os efeitos que as micelas exercem nas reações químicas tem sido estudados durante as últimas décadas, resultando no desenvolvimento de equações teóricas correspondentes aos processos químicos que ocorrem em ambientes micelares ${ }^{25-31}$.

Os sistemas micelares podem modificar a velocidade das reações e surpreende portanto, que estas características foram pouco exploradas para melhorar os métodos cinéticos de análises. As micelas catalisam as reações químicas e para que esta catálise ocorra é necessário que se cumpram duas condições:

- o substrato deve se solubilizar no agregado micelar;

- o centro da solubilização não deve impedir que o centro reativo do substrato seja acessível ao reativo atacante.

As interações eletrostáticas podem influir na etapa de transição e/ou na concentração dos reativos nas proximidades do centro da reação. Assim, uma micela catiônica pode catalisar a reação entre um ânion nucleofílico e um substrato neutro, mediante o deslocamento da carga negativa que se gera na etapa de transição e como consequência diminuir a energia de ativação da mesma. Pode também catalisar esta reação aumentando a concentração do ânion nucleófilo na interface micela-água, perto do centro reativo do substrato. Por outro lado, as interações do tipo hidrofóbico são especialmente importantes já que determinam a localização do substrato ao solubilizar-se na micela, assim como o grau de incorporação do mesmo (Figura 4.).

Deve-se ter em conta que se o substrato contém grandes grupos hidrófobos em sua estrutura, o que pode levar à formação de micelas mistas com o agente tensoativo. Este fenômeno também afeta em grande extensão a velocidade e a estereoquímica da reação na qual o substrato participa. 
Tabela 2. Comparação das características de diferentes tipos de agregados formados com o agente tensoativo.

\begin{tabular}{|c|c|c|c|c|c|c|}
\hline Características & Micelas & Micelas Inversas & Micro Emulsões & Monocapas & Bicapas & Vesiculas \\
\hline Constituinte & Tensoativo & Tensoativo & $\begin{array}{c}\text { Tensoativo, co-tensoativo, } \\
\text { solvente apolar }\end{array}$ & Tensoativo & $\begin{array}{l}\text { Tensoativo com } \\
\text { duas caudas }\end{array}$ & $\begin{array}{l}\text { Tensoativo com } \\
\text { duas caudas }\end{array}$ \\
\hline $\begin{array}{l}\text { Método de } \\
\text { preparação }\end{array}$ & $\begin{array}{l}\text { dissolver o tensoativo } \\
{[>\mathrm{CMC}] \text { em água }}\end{array}$ & $\begin{array}{l}\text { Dissolver o tensoativo } \\
\text { em sovente apolar }\end{array}$ & $\begin{array}{l}\text { Dissolver o tensoativo } \\
\text { e co-tensoatico numa } \\
\text { mistura de solventes } \\
\text { apolar-água }\end{array}$ & $\begin{array}{l}\text { Dissolver os tensoativos } \\
\text { livres em solvente } \\
\text { orgânico volátil sobre a } \\
\text { superfície aquosa }\end{array}$ & $\begin{array}{l}\text { Dissolver os tensoativos } \\
\text { livres em solvente } \\
\text { orgânico sobre um } \\
\text { furo que conecta } \\
\text { duas soluções aquosas }\end{array}$ & $\begin{array}{l}\text { Submetendo a } \\
\text { solução } \\
\text { a ultrasom }\end{array}$ \\
\hline Estrutura & 3 & & & - & 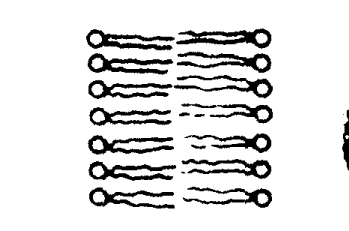 & \\
\hline $\begin{array}{l}\text { Peso molecular } \\
\text { médio }\end{array}$ & $2000-6000$ & $2000-6000$ & $10^{5}-10^{6}$ & $\begin{array}{c}\text { Depende da área } \\
\text { coberta e da densidade } \\
\text { da capa formada }\end{array}$ & $\begin{array}{l}\text { Depende da área } \\
\text { e da densidade coberta } \\
\text { da bicapa formada }\end{array}$ & $>10^{7}$ \\
\hline Diametro (A) & $30-60$ & $40-80$ & $50-100$ & $\phi$ & $\phi$ & $300-10000$ \\
\hline Estabilidade & Semanas, meses & Semanas, meses & Semanas, meses & Horas, dias & Horas & Semanas \\
\hline Diluição em água & Sào destruidas & $\begin{array}{l}\text { Formam micro } \\
\text { emulsões aq/o }\end{array}$ & $\begin{array}{l}\mathrm{o} / \mathrm{aq}+\text { água }= \\
\text { micelas aquosas }\end{array}$ & São destruidas & São destruidas & Não se alteram \\
\hline & & & $\begin{array}{c}\text { aq/o + água }= \\
\text { separação de fases }\end{array}$ & & & \\
\hline
\end{tabular}

Extraída da Tese de Doutoramento de Dolores Cecília Criado da Universidade de Córdoba 


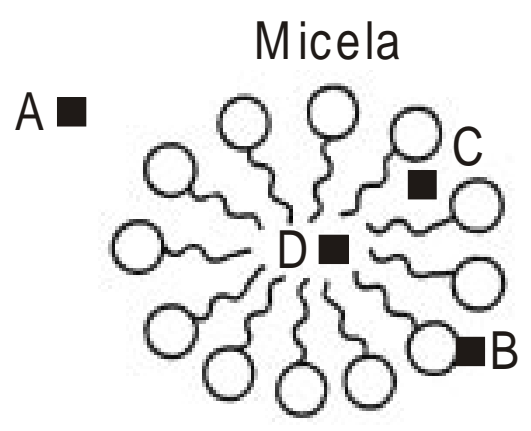

Figura 4. A, B, C e D indicam as possibilidades de interação do substrato e a micela.

Os mecanismos que envolvem esta modificação no meio reacional são complexos, e não totalmente estabelecidos, sendo que várias teorias descrevem o que pode ocorrer durante este processo ${ }^{25-31}$.

\section{Aplicações analíticas}

$\mathrm{O}$ uso de micelas oferece interessantes perspectivas na área dos métodos cinéticos de análises. Considerando as aplicações já descritas sobre a utilização de agregados micelares em diferentes campos da Química Analítica ${ }^{3,21}$ e tendo em conta os estudos físico-químicos teóricos sobre catálise micelar, o uso de micelas pode ajudar no desenvolvimento de novos métodos ou na modificação dos métodos cinéticos já existentes. Alguns dos problemas que, em princípio, podem ser solucionados são os seguintes: insolubilidade dos reativos que participam na reação, reações analíticas lentas, presença de reações colaterais, sensibilidade e/ou seletividade insuficientes, etc. É portanto surpreendente que até agora poucas aplicações tenham sido descritas, a maioria delas restritas a métodos de equilíbrio.

Dentre estas características, podem ser citados alguns trabalhos que empregam tensoativos para catalisar reações. Um exemplo refere-se ao método espectrofotométrico para a determinação simultânea de misturas binárias de cianeto, sulfito e sulfato mediante a reação com o ácido 5,5- ditobis (2-nitrobenzóico) em presença de micelas catiônicas de CTAB. A presença das micelas incrementam a diferenciação das velocidades reacionais envolvidas, permitindo dessa forma a análise ${ }^{31}$.

A catálise micelar também tem sido utilizada para melhorar os métodos espectrofotométricos para a determinação do íon cianeto baseados nas reações deste íons com dissulfetos aromáticos, tais como 5,5 ditio-bis-(2-ácido nitrobenzóico) e 4,4ditiopiridina $^{32}$. Os produtos de reação são tiocianato orgânico e o ânion tiol, sendo a absorbância do ânion formado proporcional à concentração do íon cianeto. O problema principal destes métodos é a lentidão das reações consideradas, requerendo-se de 90 a 120 minutos para se alcançar um valor constante de absorbância. O uso de micelas catiônicas de CTAB aceleram a reação e as medidas podem realizar-se depois de 1 a 2 minutos da mistura dos reativos ${ }^{33}$. É interessante destacar que a sensibilidade e seletividade dos métodos não diminui pela adição do agente tensoativo.

Dentro deste contexto, situa-se também a determinação fluorimétrica de cianeto baseado no seu efeito catalítico sobre a reação de oxidação do pirodoxal-5-fosfato pelo oxigênio dissolvido nas soluções. A adição do tensoativo DTAB ${ }^{34}$ produz um aumento do sinal de fluorescência (ao redor de 100\%) do produto formado 4-piridoxi ácido 5-fosfato, o que permite sua determinação com maior sensibilidade.

Outra importante aplicação de micelas está no emprego em reações derivativas (pré-coluna ou pós-coluna) em cromatografia líquida de alta resolução combinada com detecção por fluorescência. Um exemplo é o uso de micelas de Triton
X-100 visando derivações pré-coluna, envolvendo ácidos carboxílicos em matrizes biológicas em presença do reativo fluóforo 4-bromometil-7-metoxicumarina. Esta reação derivativa não se produz em solução aquosa devido à solvatação do grupo carboxilíco do analito. A adição do tensoativo iônico Triton X-100 permite a extração do analito na fase micelar, possibilitando que o ácido carboxílico penetre até ao coração micelar, onde se solubiliza o reativo fluoróforo; para isto é necessário se adicionar um reativo lipofílico, brometo de tetrahexilamônio, que forma um par iônico com o analito ${ }^{35}$.

A importância da catálise micelar no desenvolvimento dos métodos cinéticos tem sido preconizada por diferentes autores $^{36,37}$. Contudo, poucos estudos foram realizados até esta data neste sentido, apesar dos resultados promissores obtidos.

$\mathrm{O}$ efeito da concentração dos reativos na pseudofase micelar permitiu a aplicação de um método cinético para a determinação de aminas, fenóis, tióis, hidrazinas, mediante sua reação com 1-fluor-2,4-dinitrobenzeno (FDNB), utilizando eletrodo seletivo para quantificar os íons fluoretos liberados ${ }^{38}$. Estas reações de substituição nucleofílica são muito lentas em solução aquosa, contudo a adição de tensoativos catiônicos e não iônicos produzem uma importante aceleração das mesmas, tornando possível seu uso para determinações cinéticas. Ainda, compostos aminados tais como celafexin, sulfametiazol e vários aminoácidos tem sido determinados ${ }^{38}$.

A relação entre as constantes de velocidade e a concentração de tensoativo sugere que a interação entre a micela e os aminoácidos ocorre através da parte lipofílica e do grupo carboxílico da molécula, permitindo que o grupo amino livre reaja com $\mathrm{FDNB}^{39}$. A presença de CTAB produz um aumento de 16 vezes na velocidade de reação no caso mais favorável (triptofano). Este efeito acelerador proporcionado pelas micelas de CTAB foi utilizado também para a determinação cinética de compostos fenólicos ${ }^{38}$ e de drogas tais como a isoniazida utilizando a técnica de parada de fluxos ${ }^{40}$.

Os efeitos micelares sobre a cinética reacional também podem ser aplicados em análises de multicomponentes. Desta forma, a presença do tensoativo Triton X-100 no meio reacional permitiu a determinação simultânea de $\mathrm{Ni}(\mathrm{II})$ e de $\mathrm{Co}(\mathrm{II})$ usando 5octiloximetil-8quinolinol como reativo comum. Ambos os ions metálicos formam complexo com este reativo, sendo a velocidade de reação excessivamente alta para o seu controle mediante técnicas convencionais do monitoramento de reações rápidas. As velocidades de ambas as reações são inibidas pelo tensoativo Triton $\mathrm{X}-100$, tornando possível o acompanhamento espectrofotométrico das mesmas mediante a técnica de parada de fluxos.

\section{Pré-concentração em ambiente micelar empregando a metodologia de extração por "cloud point"}

Os métodos empregados para determinação de espécies químicas que ocorrem em baixas concentrações utilizam em geral etapas de concentração e/ou separação prévias à determinação. Os processos mais comumente empregados para esta finalidade são a extração líquido-líquido ${ }^{41,42}$ e a troca iônica, em geral envolvendo sorventes sólidos/resinas de troca iônica ${ }^{43-47}$. Contudo, são conhecidas as vantagens e as limitações que tais procedimentos possuem quanto ao seu emprego ${ }^{42,47}$.

Nos últimos anos os ambientes organizados (micelas normal ou reversa, microemulsões, vesículas, etc.) tem sido empregados em praticamente todo o campo da Química Analítica na melhoria de métodos já existentes e/ou no desenvolvimento de novas metodologias, incluindo uma particular aplicação do meio organizado em vários processos de separação. Como exemplo podem ser citadas as aplicações em HPLC, extração, filtração com gel, ultra-centrifugação, cromatografia com eletroforese capilar, as quais tem mostrado novas possibilidades de pesquisa na separação das moléculas em diversas áreas como biotecnologia, saúde pública ou no estudo de poluentes ambientais ${ }^{48-50}$. 
Tensoativos catiônicos, não iônicos e anfóteros, quando empregados em quantidades acima da concentração micelar crítica e aquecidos a uma determinada temperatura, podem separar-se em duas fases isotrópicas, fenômeno este denominado "cloud point" $48,49,51-53$.

A definição de "cloud point" está sujeita a várias interpretações muito semelhantes, que se completam. É a separação de duas fases, que parece estar associada à existência de micelas compostas por moléculas gigantes na solução. Em soluções aquosas, o emprego de tensoativos não iônicos e anfóteros formam uma fase complexa e particularmente propensa a separar-se sob uma determinada temperatura. Certos tensoativos anfóteros e alguns não iônicos em presença de altas concentrações de eletrólitos (soluccões salinas) podem também apresentar separação de fases ${ }^{49,51-55}$.

Em soluções aquosas, os tensoativos não iônicos e anfóteros com concentrações acima da CMC podem ser empregados como extratores, pois nestas soluções quando aquecidas (a temperatura depende do tensoativo empregado) duas fases distintas aparecem, sendo uma delas a fase aquosa, contendo pequena quantidade de tensoativo, abaixo da CMC e a outra, extremamente concentrada em tensoativo e contendo os componentes extraídos da solução. Quando vários tensoativos não iônicos e anfóteros em solução aquosa são aquecidos acima de uma determinada temperatura, a solução começa subitamente a ficar turva, devido ao decréscimo de solubilidade do tensoativo em água, e as fases se separam ${ }^{49,51-55}$.

Conclui-se então que a separação de fases ocorre em duas partes distintas, uma pobre e a outra rica em tensoativo contendo o analito. O mecanismo segundo o qual ocorre a separação, foi bastante investigado nas décadas de 60 a $80^{56-58}$, porém o mesmo continua sendo uma fonte de controvérsia.

Os principais fatores que evidenciam a importância do emprego de tensoativos para pré-concentrar e/ou separar em comparação a outras técnicas foram discutidos ${ }^{49,51-58}$ e os seguintes aspectos devem ser considerados:

- O tensoativo deve possuir a capacidade natural de extrair somente o analito na fase rica com altos fatores de préconcentração; o mesmo pode ser extraído em pequenos volumes de fase rica, geralmente 0,2 a $0,4 \mathrm{~mL}$, o que no mínimo indica um fator de pré-concentração idêntico às outras técnicas;

- A separação das fases depende da hidrofobicidade do analito, permitindo o uso de várias estratégias como, por exemplo, da extração líquido-líquido;

- Os tensoativos empregados não são tóxicos, são de menor periculosidade quando comparados aos solventes orgânicos usualmente empregados em extrações líquido-líquido, não são voláteis nem inflamáveis e requerem poucos miligramas sendo que os preços dos tensoativos comercialmente disponíveis são geralmente baixos;

- O processo de separação das fases é reversível.

Com relação à extração por "cloud point", Watanabe e Tanaka ${ }^{59}$ foram os primeiros a desenvolver o processo usando a indução da temperatura na separação de fases para concentrar complexos metálicos pouco solúveis em água. Estes eram solubilizados pelas micelas e concentrados com rapidez e eficiência, devido ao fato de o volume final da fase rica em tensoativo ser muito pequeno. Portanto, o uso da indução da separação de fases por temperatura melhora a sensibilidade dos métodos analíticos convencionais principalmente para íons metálicos ${ }^{53,5}$. Hoshino e colaboradores ${ }^{60}$ comentaram que as determinações de complexos metálicos em solução aquosa baseadas no emprego de reagentes orgânicos apresentam problemas indesejáveis de precipitação, que podem ser evitados pela sua solubilização nos ambientes organizados e posterior extração por separação das fases.

A separação de fases deve promover um elevado fator de concentração, propiciando melhor sensibilidade e possibilitando ainda uma melhoria em seletividade.
Neste contexto, o tensoativo polioxietileno nonil fenil eter (PONPE - 7.5) foi empregado para a determinação de zinco em águas naturais ${ }^{59}$, o qual era complexado pelo PAN e extraído da solução pelo aparecimento de duas fases à temperatura ambiente após 8 minutos de centrifugação a $4000 \mathrm{rpm}$. O fator de pré-concentração foi de aproximadamente 25 vezes e o sistema de detecção empregado era o espectrofotométrico.

Gadolinio foi espectrofotometricamente determinado em baixas concentrações $\left(\mathrm{mg} \mathrm{L}^{-1}\right)$ em amostras de urina, após formar o complexo Gd(III)-2-dichloro-2-pyridylazo)-5-dimethylaminophenol extraído e pré-concentrado pelo tensoativo não iônico PONPE-7.5 ${ }^{61}$

Recentemente, foi proposto o emprego de tensoativo aniônico na formação do "cloud point" para pré-concentrar hidrocarbonetos policíclicos (PAHs) aromáticos em água ${ }^{62}$. O uso do tensoativo aniônico ácido dodecano sulfônico (SDSA) apresenta vantagens significativas quando comparado aos tensoativos não iônicos e aos anfóteros com relação em eliminar as etapas prévias de limpeza das amostras para a determinação de PAHs.

Desta forma, cabe enfatizar que o emprego de agentes tensoativos para pré-concentrar substâncias inorgânicas mostrase um campo pouco explorado e altamente promissor. Os mesmos, na forma de complexos, tendem a solubizar-se nas micelas e podem ser separados pela metodologia de "cloud point".

\section{CONCLUSÕES}

O uso de tensoativos em Química Analítica tem se mostrado altamente promissor, uma vez que o mesmo pode propiciar um aumento de sensibilidade e/ou seletividade para um grande número de reações. Outro fator positivo esta no emprego para a pré-concentração em substituição aos solventes orgânicos pois o uso desta técnica que possui fatores de pré-concentração similares à extração líquido-líquido e também pelo fato de ser incluída na chamada "Química Verde".

\section{AGRADECIMENTOS}

O autor agradece E.A G. Zagatto pela colaboração e sugestões, D. Pérez-Bendito, da Universidade de Córdoba, Espanha por participar junto ao seu grupo de trabalho e à FAPESP pelo suporte financeiro.

\section{REFERENCIAS}

1. Weest, C. C.; Harwell, J. H.; Environ. Sci. and Techn. 1992, 26, 2324.

2. Porter, M. R.; "Recent developments in the analysis of surfactants. Critical reports on applied chemistry”, N.Y., vol. 32 Ed. Elsevier Science Ltde, 1978.

3. Hinze, W. L.; "Solution chemistry of surfactants" K.L. Mittal, N.Y, Ed Plenunn Press, 1979.

4. Pelizzetti, E.; Pramauro, E.; Anal. Chim. Acta 1985, 169, 1.

5. Quina, F. H.; Hinze, W. L.; Ind. Eng. Chem. Res. 1999, 38,4150 .

6. Saunders, L.; Biochim.Biophys. Acta 1966, 125, 70.

7. Attwood, D.; Elworthy, P. H.; Kayne, S. B.; J. Phys. Chem. 1970, 74, 3529.

8. Geer, R. D.; Eylar, E. H.; Anacker, E. W.; J. Phys. Chem. 1971, 75, 369.

9. Kurz, J. L.; J. Phys. Chem. 1962, 66, 2239.

10. Fendler, E. J.; Day, C. L.; Fendler, J. H.; J. Phys. Chem. 1972, 76, 3529.

11. Elworthy, P. H.; Florence, T. A.; Macfarlane, C. B.; "Solubilization by surface active agents and its applications in chemistry and the biological sciences", London, Ed. Chapman \& Hall, 1968.

12. Fendler, J. H.; "Membrane mimetic chemistry", N.Y., Ed. John Wiley, 1992. 
13. Preston, W. C.; J. Phys. Chem. 1948, 52, 848.

14. Rosen, M. J.; "Surfactants and interfacial phenomena", N.Y., Ed. Wiley-Interscience, 1978.

15. Fendler, J. H.; Fendler, E. J.; "Catalysis in micellar and macromolecular systems”, N.Y., Ed. Academic Press, 1975.

16. Shapiro, D.; "Chemistry of natural products" Paris, Vol IX, Ed. E. Lederer, 1969.

17. Mysels, K. J.; "Introduction to colloid chemistry”, N.Y., Ed. Wiley Interscience, 1958.

18. Tanford, C.; J. Phys. Chem. 1972, 76, 3020

19. Schott, H.; J. Pharm. Sci., 1971, 60, 1594.

20. Zografi, G.; Yalkowsky, S.H.; J. Pharm. Sci., 1972, 61, 651.

21. Mukerjee, P.; Mysels, K. J.; "Critical micelles concentration of aqueous surfactant systems”, Washington: U.S. Gov. Printing Office, Superintendent of Documents, 1971. (NSRDS-NBS 36.)

22. Pilpel, N.; Chem. Ver. 1963, 63, 221.

23. Durham, K.; "Surface activity and detergency", N.Y., Ed.Macmillan, 1961.

24. Cordes, E. H.; “Reaction kinects in micelles”, N.Y., Ed. Plenunn Press, 1973.

25. Menger, F. M.; Jerkunica, J. M.; Johnston, J. C.; J. Am. Chem. Soc. 1978, 100, 4676.

26. Menger, F. M.; Yoshinaga, H.; Venkatusubban, K. S.; J. Org. Chem., 1981, 46, 415.

27. Menger, F. M.; Nature 1985, 313, 603.

28. Rodgers, M. A. J.; Chem. Phys. Letters 1981, 78, 509.

29. Menger, F. M.; Doll, D. W.; J. Am. Chem. Soc. 1984, 106, 1109.

30. Dill, K. A.; Koppel, D. E.; Cantor, R. S.; Dill, J. D.; Bendeclouch, D.; Chen, S. H.; Nature 1984, 309, 42.

31. Cabane, B.; Nature 1985, 314, 385.

32. González, V; Moreno, B; Sicilia, D.; Rubio, S.; PerezBendito, D.; Anal. Chem. 1993, 65, 1897.

33. Love, L. J. C.; Habarta, J. G.; Dorsey, J. G.; Anal. Chem. 1984, 56, 1133.

34. Sicilia, D.; Rubio, S.; Pérez-Bendito, D.; Maniasso, N.; Zagatto, E. A. G.; Analyst 1999, 124, 615.

35. Mori, I.; Fujita, Y.; Fujita, K.; Nakahashi, Y.; Tanaka, T.; Shihara, S.; Fresenius Anal. Chem. 1988, 330, 619.

36. Van der Horst, F. A. L.; Trends Anal. Chem. 1989, 8, 268.

37. Hinze, W. L.; Singh, H. N.; Baba, Y.; Harvey, N. G.; Trends Anal. Chem. 1984, 3, 193.
38. Athanasioumalaki, E.; Koupparis, M. A.; Anal. Chim. Acta 1989, 219, 295.

39. Pérez-Bendito, D.; Rubio, S.; Anal. Chim. Acta 1989, 224, 185.

40. Archontacki, H. A.; Koupparis, M. A.; Efstathiou, C. E.; Analyst 1989, 114, 591.

41. Jungclaus, G. A.; Games, L. M.; Hites, R. A.; Anal. Chem. 1976, 48, 1894.

42. Blanco. T.; Maniasso, N.; Giné, M. F.; Jacintho, A. O.; Analyst 1988, 123, 191.

43. Basu, D. K.; Saxena, J.; Environ. Sci. Technol. 1978, 12, 791.

44. Symons, R. K.; Crick, I.; Anal. Chim. Acta 1983, 151, 237.

45. van Rossum, P.; Webb, R. G.; J. Chrom. 1979, 150, 237.

46. Inukai, Y.; Chinen, T.; Matsuda, T.; Kaida, Y.; Yasuda, S.; Anal. Chim. Acta 1998, 371, 187.

47. Xu, B. X.; Fang, Y. Z.; Talanta, 1988, 35, 891.

48. Pinto, C. G.; Pavón, J. L. P.; Cordero, B. M.; Anal. Chem. 1992, 64, 2334.

49. Laespada, M. E. F.; Pavón, J. L. P.; Cordero, B. M.; Analyst 1993, 118, 209.

50. Colambara, R.; Tavares, M. F. M.; Massaro, S.; Quim. Nova 1997, 20, 512.

51. Cordero, B. M.; Pavón, J. L. P.; Pinto, C. G.; Laespada, M. E. F.; Talanta 1993, 40, 1703.

52. Saitoh, T.; Hinze, W.; Anal. Chem. 1991, 63, 2520.

53. Saitoh, T.; Tani, H.; Kamidate, T.; Watanabe, H.; Trends in Anal. Chem. 1995, 14, 213.

54. Ferrer, R.; Beltrán, J. L.; Guiteras, J.; Anal. Chim. Acta 1996, 330, 199.

55. Sirimanne, S. R.; Barr, J. R.; Patterson Jr, D. G.; Anal. Chem. 1996, 68, 1556.

56. Corti, M.; Mimeiro, C.; Digiorgio, V.; J. Phys. Chem, 1984, 88, 309.

57. Degiorgio, V.; Piazza, R.; Corti, M.; Mineiro, C.; J. Phys. Chem. 1985, 82, 1025.

58. Goldstein, R. E.; J. Phys. Chem. 1986, 84, 3367.

59. Watanabe, H.; Tanaka, H.; Talanta 1978, 52, 585.

60. Hoshino, H.; Saitoh, T.; Taketomi, H.; Yotsuyanagi, T.; Anal. Chim. Acta 1983, 147, 339.

61. Silva, M. F.; Fernandez, L. P.; Olsina, R. A.; Analyst 1998, 123, 1803.

62. Sicilia, D.; Rubio, S.; Pérez-Bendito, D.; Maniasso, N.; Zagatto, E. A. G.; Anal. Chim. Acta 1999, 392, 29 